\title{
A Foresighted Driver Model derived from Integral Expected Risk
}

\author{
Julian Eggert $^{1}$ and Fabian Mueller ${ }^{2}$
}

\begin{abstract}
Current efforts in Advanced Driver Assistant Systems and Autonomous Driving research target at making the vehicles more intelligent, in terms of understanding what is going on and selecting the most appropriate behaviors. A crucial element of this research is the prediction of the evolution of the current driving situation with microscopic driver models. In this paper we present a microscopic driver model with a gradientlike, simple behavior generation that is fully and concisely derived from mathematical risk theory. Following this model, drivers act by estimating the expected, integral future risks and benefits and by seeking the best instantaneous tradeoff between these quantities, choosing the immediate action that reduces the hypothetical risks in the most efficient way. We show how this model is able to incorporate different risk types and situation parameters, allowing an extension and generalization to variable scenarios.
\end{abstract}

\section{INTRODUCTION}

Microscopic driver models are widely used to predict the behavior of traffic participants (TP's). Especially for current research in Advanced Driver Assistant Systems (ADAS) and Autonomous Driving (AD), it is necessary to predict where the own and the other traffic participants will go and how they will behave, see [1] for a review. With this prediction, the likely evolution(s) of the current driving scene are estimated. In a next step, a risk assessment can be performed, by estimating the criticality resp. the risk of the own predicted behavior within the predicted scene. By systematic variation of the own behavior, appropriate safe actions can be found as the result of an optimization process, see [2]-[5] for behavior architectures which follow this scheme.

Microscopic driver models describe a single traffic participants' behavior with the purpose of predicting the most likely time course of a scenario as a forward simulation of the current situation [6], [7]. For example, in the Intelligent Driver Model (IDM) ( [8] and related work) the longitudinal velocity adaptation is described by a differential equation which incentives to drive forward at the same time as keeping a certain velocity-dependent safety distance to the vehicle in front. Although very successful for the analysis of macroscopic traffic flow phenomena, the IDM is a heuristic approach with lacking systematic derivation from grounded risk theory. In addition, its applicability to complex scenarios is rather limited due to the restriction to longitudinal cases and the consideration of a single risk source. However,

\footnotetext{
${ }^{1}$ Julian Eggert is with the Honda Research Institute (HRI) Europe, Carl-Legien-Str. 30, 63073 Offenbach/Main, Germany julian.eggertahonda-ri.de

${ }^{2}$ Fabian Mueller is with the Control Methods and Robotics Lab, Technical University of Darmstadt, 64283 Darmstadt, Germany fabian.muel lerermr.tu-darmstadt. de
}

extensions of the IDM have also been applied for modeling lane-change scenarios [9] as well as speed adaptation to curvy roads and in intersections [10].

In [11], the Foresighted Driver Model (FDM) has been introduced in an attempt to overcome some of the mentioned driver model limitations. In the FDM, starting from a driving scene prediction the expected risks and benefits are estimated, and the behavior is chosen so as to minimize the the overall costs, i.e., minimizing the risks and maximizing the benefits. Risk sources and risk indicators are incorporated explicitly into the model, describing collision risks by e.g. classical risk indicators such as the time and distance of closest encounter between pairs of predicted trajectories. The explicit modeling of risk sources allowed for a greater flexibility to generalize across different scenarios, so that extensions of the FDM were applied on non-longitudinal driving situations (intersections and entrances), tactical lane change, overtaking and continuous lateral control [12]. Nevertheless, the single risks were still modeled in a rather phenomenological way, so that a derivation of a microscopic driver model from first principles of risk theory remains an open question.

To address this issue, in this paper we present an extension of the Foresighted Driver Model approach, termed FDM++, which starts from the elementary concepts of event probabilities under uncertain conditions, and in combination with the so-called survival theory [13] derives a microscopic driver model based on the consideration of risks and benefits from basic events. The survival theory has already been applied in the more complex setting of risk maps [14] to driver behavior planning in a range of different scenarios, so that we consider it as a good starting point for our approach.

In detail, the main contributions of this paper are:

1) We derive a driver model from a concise theoretical foundation for the probability of risky events under uncertainty.

2) We estimate the risk by calculating the probability when the ego-vehicle will be hit by a critical event.

3) We present scalar integral measures that serve to assess safety of he expected future of the driving scene, including its risk.

4) We derive a model for driver behavior which chooses the current action that minimizes the future integral of expected risk.

5) We show that the model is open to incorporate different types of risks and that it is applicable to longitudinal and lateral scenarios.

6) We derive a novel way of calculating future collision risks with explicit uncertainty considerations. 


\section{RISK MODEL}

We follow a classical risk modeling approach, by assuming that risk is the combination of the probability of a critical event multiplied by the cost or benefit that incurs when that event happens (see e.g. [15]). Since the events are hypothetical (as they may or may not happen in the future), and since they can happen at any time, risk is the future cost expectation value resp. the integral of the predicted event probability over all future times multiplied with the associated cost of the event at each particular time.

Let us consider a driving scene in which different risky events $e$ can possibly affect our ego-vehicle (e.g. it can potentially collide with a series of $N_{e}$ objects or other vehicles). Starting at $t$, for a particular prediction of the scene evolution along future time $t+s$, for the time interval of length $\Delta t$ around $t+s$ and for each event $e$ we then would have a risk

$$
R_{\mathrm{FE}, e}(t+s ; \Delta t):=P_{\mathrm{FE}, e}(t+s ; t, \Delta t) C_{e}\left[I_{e}(t+s)\right]
$$

given by the event probability $P_{\mathrm{FE}, e}{ }^{1}$ and the related costs $C_{e}{ }^{2}$. The total risk is then given by accumulating the risks for all future time to get the total (i.e., integral) expected cost,

$$
C(t)=\sum_{e=1}^{N_{e}} \int_{s=0}^{\infty}\left[R_{\mathrm{FE}, e}(t+s ; \Delta t) / \Delta t\right] d s .
$$

Since this quantity depends on the future scene evolution, once the total expected cost $C(t)$ can be calculated, safe driving strategies can be found by analysing the cost changes under variation of the ego-vehicle predicted trajectory. In the following sections we therefore proceed to derive $C(t)$ from the predicted scene evolution and an event-based risk estimation approach.

For this purpose, we first introduce in section III the notion of risk indicators and how to deal with different sources of risk under uncertainties in sensor measurements and prediction. From the risk indicators, in section IV first an instantaneous event probability is calculated, which serves as the basis for the so-called survival function. The survival function itself then serves to model the future behavioral uncertainty as well as the probability that a traffic participant will reach a certain point in the future predicted trajectory. In section $\mathrm{V}$, using the survival function we then derive integral risk measures which serve to quantify the criticality as well as the fitness of purpose of the predicted trajectories. In section VI, we describe the complete FDM++ model and in section VII we show exemplary simulations based on the model.

\footnotetext{
${ }^{1} \mathrm{FE}$ stands for "First Event", meaning that $P_{\mathrm{FE}, e}$ is the probability that event $e$ takes place for the first time at $t+s$ if we start at $t$. We will come back to this formulation later in sections IV-B and V-A.

${ }^{2} I_{e}$ are the risk indicators resp. the predicted scene parameters relevant for the cost calculation when event $e$ happens, e.g. the speed, proximity, angle of impact and masses of the involved vehicles.
}

\section{SOURCES OF RISK AND RISK INDICATORS}

In a normal driving scene, several different risk types can occur, caused by many risk sources with each leading to one or more possible risk events, with each event $e$ characterized by the so-called risk indicators $I_{e}$. The main sources of risk are e.g. other traffic participants, and the risk types can be roughly categorized into collision with other vehicles (risk indicators proximity, velocity differences, relative orientations), collision with vulnerable traffic participants like pedestrians, collision with road environment, and risk emanating from driving dynamics and loss of control (e.g. curve risk with risk indicators curvature, velocity, friction coefficient, etc.).

The risk indicators are inherently noisy, since they come either from noisy sensory measurements or they are the result of the trajectory prediction which comprises an uncertainty over the future state prediction. Since our target is a driving model which takes near-optimal behavioral decisions even in situations with partial sensory and evolution uncertainty, we introduce minimal models for uncertainty parametrization.

In particular, for the case of collision risks, the uncertainty in the position of the traffic participants is the most important factor. Here, as a rough approximation, we model the position uncertainty as a 2D Gaussian distribution of the position probability, around a mean position $\hat{\mathbf{x}}$. This serves both for the description of the current position, as well as for the future positions during the prediction of the likely trajectories of the traffic participants ${ }^{3}$. For the current positions of other TP's, the Gaussian distributions serve to model the combined measurement and state evolution uncertainty; for the future positions they model a mixture between measurement and estimation uncertainty and further uncertainty propagation caused by behavioral uncertainty of the other TP's.

\section{A. Uncertainty over Future Position of Traffic Participants}

For simplicity we work in the Frenet frame, separating longitudinal and lateral positional components relative to the driving path. For the longitudinal component, for a single traffic participant we assume its real position to be centered around $\hat{x}(t)$ according to:

$$
p(x ; t):=\frac{1}{\sqrt{2 \pi \sigma_{x}(t)^{2}}} \exp \left\{-\frac{1}{2} \frac{[x-\hat{x}(t)]^{2}}{\sigma_{x}(t)^{2}}\right\} .
$$

In addition we assume the longitudinal velocity to be also distributed around a velocity $\hat{v}$ :

$$
p(v ; t):=\frac{1}{\sqrt{2 \pi \sigma_{v}(t)^{2}}} \exp \left\{-\frac{1}{2} \frac{[v-\hat{v}(t)]^{2}}{\sigma_{v}(t)^{2}}\right\} .
$$

For the trajectory prediction from time $t$ into the future $t+s$, we then assume the future positions to be centered

\footnotetext{
${ }^{3}$ Other distributions, like the Kumaraswamy distribution [16], might be more accurate in describing future position probabilities e.g. because they have lower and upper bounds, however Gaussian distributions suffice for our modeling purpose and are easier for calculations.
} 


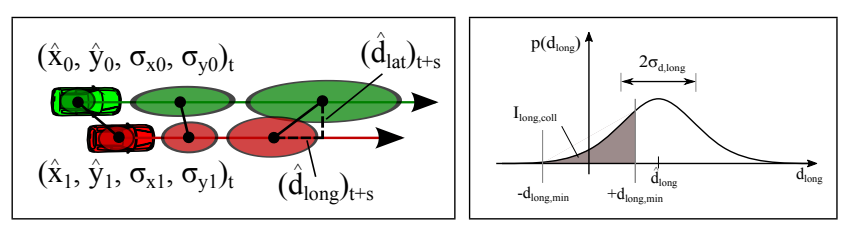

Fig. 1: Prediction of positional uncertainty by Gaussian spatial distributions for ego and other vehicle (left) and calculation of collision probability indicator function from the overlap of the two distributions at a particular point in time point

around longitudinal future mean positions $\hat{x}(t+s)$ with future variances $\sigma(t+s)$

$p(x ; t, s):=\frac{1}{\sqrt{2 \pi \sigma_{x}(t+s)^{2}}} \exp \left\{-\frac{1}{2} \frac{[x-\hat{x}(t+s)]^{2}}{\sigma_{x}(t+s)^{2}}\right\}$.

The future variance is gained from the consideration that a vehicle with initial velocity $\hat{v}(t)+\Delta v(t)$, will have a future position $x(t+\Delta t)+\Delta v(t) \Delta t$, so that for the positional variance caused by the velocity variance it holds that $\sigma_{x, v}(t+$ $\Delta t)=\sigma_{v}(t) \Delta t$.

With a longitudinal velocity variance which increases with higher mean velocity i.e., $\sigma_{v}(t)=\alpha_{v} \hat{v}(t)$, for positive velocities we set

$$
\sigma_{x, v}(t+s)=\int_{0}^{s} \alpha_{v} \hat{v}(t+s) \mathrm{d} s=\alpha_{v}[\hat{x}(t+s)-\hat{x}(t)]
$$

for the longitudinal positional variance increase caused by the velocity distribution. In addition, at time $t$ we have a noisy position measurement expressed by a variance $\sigma_{x, p}(t)$ which adds additional uncertainty. Combining the uncertainties in position from measurement and velocity variation we then get the future positional uncertainty for the predictions according to

$$
\left[\sigma_{x}(t+s)\right]^{2}=\left[\sigma_{x, p}(t)\right]^{2}+\left[\sigma_{x, v}(t+s)\right]^{2},
$$

which we use in Eq. (5). The left side of Fig. 1 schematically shows the variance increase over prediction time.

\section{B. Prediction of Collision Probability}

Considering now two vehicles with longitudinal position probabilities $p\left(x_{0}\right)$ and $p\left(x_{1}\right)$, we get that the probability distribution of their (signed) distance $d_{\text {long }}:=x_{1}-x_{0}$ is (by convolution theorem)

$$
p\left(d_{\text {long }}\right):=\frac{1}{\sqrt{2 \pi \sigma_{d_{\text {long }}}^{2}}} \exp \left\{-\frac{1}{2} \frac{\left[d_{\text {long }}-\hat{d}_{\text {long }}\right]^{2}}{\sigma_{d_{\text {long }}}^{2}}\right\}
$$

with

$$
\hat{d}_{\text {long }}=\hat{x}_{1}-\hat{x}_{0} \text { and } \sigma_{d_{\text {long }}}^{2}=\sigma_{x_{0}}^{2}+\sigma_{x_{1}}^{2} .
$$

The future (i.e. predicted) distance probability distribution $p\left(d_{\text {long }} ; t, s\right)$ is calculated likewise using the predicted $\hat{x}_{0}(t+$ $s), \hat{x}_{1}(t+s)$ and the $\sigma_{x_{0}}(t+s), \sigma_{x_{1}}(t+s)$ from Eq. (7).

The two vehicles will overlap if their distance is smaller than their physical longitudinal extension, given by $d_{\text {long,min }}$.
We therefore have that $p\left(d_{\text {long }}\right)$ is 1 if $\left|d_{\text {long }}\right| \leq d_{\text {long,min }}$ and 0 elsewhere, so that a risk indicator function for collision by spatial overlap is given by (see right side of Fig. 1 for an explanation):

$$
I_{\text {long, }, \text { oll }}:=\int_{-d_{\text {long,min }}}^{+d_{\text {long,min }}} p\left(d_{\text {long }}\right) \mathrm{d} d_{\text {long }}
$$

Substituting, after some calculations we get

$$
I_{\text {long,coll }}=\frac{1}{2}\left\{\operatorname{erf}\left[\frac{d_{\text {long,min }}-\hat{d}_{\text {long }}}{\sqrt{2} \sigma_{d_{\text {long }}}}\right]-\operatorname{erf}\left[\frac{-d_{\text {long,min }}-\hat{d}_{\text {long }}}{\sqrt{2} \sigma_{d_{\text {long }}}}\right]\right\}_{\text {(11) }} .
$$

In the limiting case $d_{\text {long,min }} \rightarrow \infty$ or $\sigma_{d_{\text {long }}} \rightarrow 0$, it is $I_{\text {long,coll }} \rightarrow$ 1 as intuition would tell.

These considerations work for longitudinal settings and can be extended to scenarios with parallel lanes, as e.g. for two-lane opposite direction traffic or multilane runways by introducing a similar probability distribution for the lateral distance. Starting from general 2D coordinates $\mathbf{x}$ and position probability distributions $p(\mathbf{x})$, transforming everything into the path-relative coordinate system we get that $p(\mathbf{x})$ factorizes multiplicatively into longitudinal and lateral components, and so does the 2D overlap probability calculated from the distributions, leading to $I_{\text {long,coll }}$ with $d_{\text {long,min }}$ and $I_{\text {lat,coll }}$ with $d_{\text {lat,min }}$. Finally, we get that

$$
I_{\text {coll }}=I_{\text {long,coll }} I_{\text {lat,coll }} .
$$

In this way, using predicted positional means and variances (i.e., $\hat{x}(t+s), \sigma_{x}(t+s)$, etc.), we can calculate the future 2D expected overlap probability in an analytic, cheap way, using the future positional probability distributions.

For non-longitudinal settings such as intersections and lane changes, the approach can be generalized to oriented Gaussian distributions in $2 \mathrm{D}$, without however leading to analytical solutions but requiring numerical integration to calculate the overlap probability by means of the distribution overlap [17]. Collision risks with pedestrians or with environment structures can be handled in analogous way, by adaptation of the prediction model parameters for the positional distributions.

\section{SURVIVAL THEORY}

\section{A. Instantaneous Event Probability}

Traffic participants get involved in critical events, like accidents, in a way that correlates with their net instantaneous event rate $\tau^{-1} \in\left[0, \tau_{\max }^{-1}\right]$, i.e., the number of events that occur per unit time interval for a certain type of situations. In a statistical setting, if situations get more dangerous, the probability of events resp. the average number of events per time increases. We therefore have that the probability of a critical event in the time interval $[t, t+\Delta t]$ is

$$
P_{\mathrm{E}}(t ; \Delta t)=\tau^{-1}[I(t)] \Delta t
$$

with $I$ being the situation-related risk indicator function which increases monotonously with risk and $\Delta t$ the measurement time interval length. For example, if we consider 
the collision event between two specific vehicles, for $I(t)$ we use the collision probability function so that $I=I_{\text {coll }}$ from section III-B.

The instantaneous event rate is then modeled as a simple nonlinear threshold process where the instantaneous event rate increases in correlation with the risk indicator function. All events of the same type of risk, e.g. collision risks between vehicles, usually share the same risk indicator function. For $\tau^{-1} \in\left[0, \tau_{\max }^{-1}\right]$ and a risk indicator function $I \in[0,1]$ like the overlap probability from section III-B, an increasing instantaneous event rate function with adjustable slope $\beta$ is given by

$$
\tau^{-1}[I]:=\tau_{\max }^{-1}\left[\frac{1-e^{-\beta I}}{1-e^{-\beta}}\right] .
$$

However, for each type of risk, there are different possible critical events. For example, between an ego vehicle and $N_{e}$ other vehicles in the vicinity, by comparing the positions of the ego vehicle with each other vehicle there are $N_{e}$ possible sources of risky events, since the ego vehicle can collide with each of the other vehicles. Each risk source $e$ has its own event probability $P_{\mathrm{E}, e}(t)$ and its own risk indicator function $I_{e}(t)$ with parameters. In the collision risk event case, these parameters would be the expected mean positions and position variances of the involved traffic participants.

\section{B. Events over Time: Survival Function}

In the previous section, we have seen that a vehicle can be engaged in any of a large number of possible critical events $e$. This leads to event probabilities

$$
P_{\mathrm{E}, e}(t ; \Delta t)=\tau_{e}^{-1}\left[I_{e}(t)\right] \Delta t
$$

The probability that the vehicle is not engaged in any of the events in the next time interval, i.e., that the vehicle survives, is then given by

$$
P_{\mathrm{S}}(t+\Delta t, t)=\prod_{e}\left[1-P_{\mathrm{E}, e}(t ; \Delta t)\right]
$$

which for sufficiently small $P_{\mathrm{E}, e}(t ; \Delta t)$ (fulfilled for small time intervals $\Delta t$ ) amounts to

$$
P_{\mathrm{S}}(t ; \Delta t) \approx 1-\sum_{e} P_{\mathrm{E}, e}(t ; \Delta t)=1-\underbrace{\sum_{e} \tau_{e}^{-1}\left[I_{e}(t)\right]}_{:=\tau_{\mathrm{tot}}^{-1}(t)} \Delta t
$$

with the total event rate $\tau_{\text {tot }}^{-1}$ given by the sum of the single event rates. In short, the vehicle survives if it is not engaged in one event and not engaged in another event, and so on.

With this in mind, we can calculate the time course of the surviving probability of a vehicle by repeatedly applying Eq. (16). Given that it survived until time $t$ with probability $P_{\mathrm{S}}(t)$, we get

$$
P_{\mathrm{S}}(t+\Delta t)=\left[1-\tau_{\mathrm{tot}}^{-1}(t) \Delta t\right] P_{\mathrm{S}}(t)
$$

For small time intervals $\Delta t \rightarrow 0$ we get by integration the solution

$$
P_{\mathrm{S}}(t+s)=\underbrace{\exp \left\{-\int_{0}^{s} \tau_{\mathrm{tot}}^{-1}\left(t+s^{\prime}\right) \mathrm{d} s^{\prime}\right\}}_{:=S(t+s, t)} P_{\mathrm{S}}(t)
$$

with the so-called Survival Function $S(t+s, t)$ which indicates the probability that a vehicle which was ok at time $t$ has not yet engaged in any event until time $t+s$.

If $t$ is the current and $s$ the future time, the survival function accounts for the probability that a traffic participant will not get involved in any of the critical events in the future time course of predicted evolution of a driving scene. The survival function is always decreasing, from 1 to 0 for $s \rightarrow \infty$. However, depending on the occurring risks, expressed by the predicted, instantaneous event rates as a function of future time, it might decrease faster and exhibit steps at particular critical points in time.

\section{Risk Measures and Survival Probability}

\section{A. Probabilities of Predicted Events}

The survival function is an account for the time course of the risk that a vehicle undergoes during the time period $[t, t+s]$, with $s$ in the range of several seconds. It can be used for a series of further very useful analyses of the criticality that a vehicle is exposed to. If we are interested in the overall probability that a particular critical event $e$ happens exactly at time $t+s$ in the future, e.g. the collision with a specific other vehicle, this is equivalent to the fact that the ego-vehicle will survive until $t+s$ and then be engaged in this event for the first time in the time interval of length $\Delta t$ around $t+s$, so that

$$
P_{\mathrm{FE}, e}(t+s ; t, \Delta t)=P_{\mathrm{E}, e}(t+s ; \Delta t) S(t+s, t) .
$$

This can of course happen over the entire predicted future. Accumulating the probabilities over all future times we get

$$
P_{\mathrm{INT}, e}(t)=\int_{0}^{\infty} \tau_{e}^{-1}\left[I_{e}(t+s)\right] S(t+s, t) \mathrm{d} s
$$

where we used Eq. (15) in combination with the survival function from Eq. (19). The gained result is the overall timeintegrated expected probability (starting prediction at $t$ ) that the ego-vehicle will be engaged in the event of risk source $e$ from $t$ on.

It is a true probability over the risk events, in the sense that

$$
P_{\mathrm{INT}}(t):=\sum_{e} P_{\mathrm{INT}, e}(t)=1
$$

i.e. at any moment in time, if we look at all possible events that the ego-vehicle can potentially be engaged in, over the entire predicted future, the ego-vehicle will be hit by one of the events for certain ${ }^{4}$.

\footnotetext{
${ }^{4}$ Intuitively, this corresponds to the saying: "On the very long run, we certainly end up dead."
} 


\section{B. Escape Probability}

The normalization leads to an important property of the approach. We consider an event to be anything disruptive that can happen in the predicted future which leads to a deviation from an assumed trajectory, so that the predicted evolution of the situation becomes invalid. Most of these events are caused by true risks associated with possible costs, damage, or injuries. However, a deviation from an assumed trajectory can also occur by erratic behavior, e.g. some random steering or acceleration variations, which move the vehicle away from the assumed path, etc. These are the so-called "escape events", for which we reserve the first index $e=0$. These events are of uncertain origin, and we model them by an "escape rate" $\tau_{0}^{-15}$.

Then, the probability of escaping over the entire predicted future of an assumed trajectory is given by

$$
P_{\mathrm{INT}, o}(t)=\tau_{0}^{-1} \int_{0}^{\infty} S(t+s, t) \mathrm{d} s .
$$

As a consequence, without any further risks, the survival function $S(t+s, t)$ decays exponentially with $\tau_{0}^{-1}$, meaning that trajectory states further in the future are less likely to be reached, introducing a finite prediction time horizon. Second, risks which are very close in terms of prediction time have greater influence, whereas risks further away in the future are discounted by the survival function. And third, it is $P_{\mathrm{INT}, o}(t)=1-\sum_{e=1}^{N_{\mathrm{E}}} P_{\mathrm{INT}, e}(t)$, i.e., the probability to escape is equal to the probability of not getting engaged in any of the hypothetical future risks.

\section{Integral Instantaneous Future Expectations}

Using the survival function, we can calculate very useful trajectory quantifiers by integrating some function over the entire prediction time interval:

$$
F_{\mathrm{INT}}(t):=\langle F(t+s)\rangle_{\mathrm{s}}:=\int_{0}^{\infty} F(t+s) S(t+s, t) \mathrm{d} s .
$$

These serve to quantify expectation values (e.g. costs and benefits) along a predicted trajectory. The expectation values take into account the natural prediction horizon given by the survival function. As an example, if we want to estimate expected driving benefits, Eq. (24) only integrates them on the trajectory portion which we are certain to drive ${ }^{6}$.

E.g. from Eqs. (21) and (23), we have seen how to integrate appropriately over the risk rates,

$$
\begin{aligned}
& P_{\mathrm{INT}, e}(t)=\left\langle\tau_{e}^{-1}\left[I_{e}(t+s)\right]\right\rangle_{\mathrm{s}} \\
& P_{\mathrm{INT}, o}(t)=\left\langle\tau_{0}^{-1}\right\rangle_{\mathrm{s}}
\end{aligned}
$$

which gave us scalar, integral risk event probabilities $\in[0,1]$ for each risk source, and which are good measures for the future time-integrated risk given an assumed scene evolution, as well as the escape probability.

\footnotetext{
${ }^{5}$ The term "escape" expresses that the vehicle will as consequence avoid, i.e., "escape from" all subsequent risks after such an event happens.

${ }^{6}$ This is a necessary condition for sensible trajectory evaluation statistics, since an integration without survival function would always scale with trajectory length and additionally lead to a disproportional weight of contributions that lie far ahead in the prediction future.
}

\section{Future Expected Risk}

We have so far seen how to calculate sensible expectation values for the probability of critical events. Nevertheless, risk is not equivalent to event probability. Rather, it is a combination of the risk event probability $P_{E}$ with its severity, e.g. given by the incurred cost $C_{e}{ }^{7}$ if the event happens [18]. This means that an instantaneous risk is given by

$$
R_{e}(t ; \Delta t)=\tau_{e}^{-1}\left[I_{e}(t)\right] \Delta t C_{e}\left[I_{e}(t)\right]:=r_{e}(t) \Delta t
$$

with the differential risk (risk per time unit) $r_{e}$. (For simplicity, here we have assumed that the incurred cost depends on the same state parameters as the event rates.)

The integral expected risk for each event is then given by

$$
R_{\mathrm{INT}, e}(t)=\left\langle r_{e}(t+s)\right\rangle_{\mathrm{s}} .
$$

The event costs are accumulated by adding them over all possible events to an overall integral risk

$$
R_{\mathrm{INT}}(t)=\sum_{e=1}^{N_{e}} R_{\mathrm{INT}, e}(t),
$$

where the escape events $(e=0)$ are omitted since they are not related to a risk event but rather describe deviating driving behavior that leads to a change in trajectory and therefore to an avoidance of the following risks (as they do not lead to any damage, the escape events are considered to have zero event costs $C_{0}=0$ ).

\section{E. Future Expected Utility}

Similarly to the risk, we can proceed with continuous (i.e., non-event driven) driving qualities, such as other costs and utilities not related to risky events, and which incur during the driving process. Let us say that there are $u$ different driving qualities, expressed by cost functions $q_{u}$. Then we get that the cost for each driving quality is given by

$$
Q_{\mathrm{INT}, \mathrm{u}}(t)=\left\langle q_{u}(t+s)\right\rangle_{\mathrm{s}}
$$

and, correspondingly, the total driving quality related costs amount to

$$
Q_{\mathrm{INT}}(t)=\sum_{u=0}^{N_{u}} Q_{\mathrm{INT}, \mathrm{u}}(t) .
$$

One straightforward example is the transportation gain, which can be expressed by using the driving distance that the vehicle is expected to drive under the given risk conditions, i.e., while it keeps "surviving" and driving along the predicted trajectory. Since the driven distance is gained by integrating the longitudinal velocity over time, we get

$$
Q_{\mathrm{INT}, \text { trans }}(t)=-m_{\text {trans }}\langle v(t+s)\rangle_{\mathrm{s}},
$$

where $m_{\text {trans }}$ is the associated cost benefit in units of money per traveled distance. The integral term $\langle\ldots\rangle_{\mathrm{S}}$ delivers the expected distance that the vehicle will continue to drive given the current risks. The transportation gain has a double effect:

\footnotetext{
${ }^{7}$ Typical cost terms for severity are given e.g. by $C_{e}[I] \propto k_{0}+k_{1} v_{0}^{2}+$ $k_{3}\left(v_{0}-v_{e}\right)^{2}$, but this is not at the focus of the derivation of this paper.
} 
Without further constraints, it will consider those trajectories as beneficial which move the vehicle forward as fast and as long as possible. It leads to a preference of long survival times resp. slowly decaying survival functions, avoiding risks that are close ahead in time.

While $Q_{\mathrm{INT}, \mathrm{rrans}}$ is useful for nearly all driving scenarios, some of the driving quality cost factors apply selectively. E.g. for driver models it is often assumed that there is a preferred cruising velocity to which drivers adhere in case of unperturbed driving. This preference can be included by an additional driving quality cost

$$
Q_{\mathrm{INT}, \text { cruise }}(t)=m_{\text {cruise }}\left\langle\left\|v(t+s)-v_{\text {cruise }}\right\|^{2}\right\rangle_{\mathrm{s}} .
$$

A speed limit can be enforced in a similar way, by an asymmetric velocity-dependent cost term. Further useful driving quality measure include e.g. the penalization of large accelerations $a$ by

$$
Q_{\mathrm{INT}, \text { comfort }}(t)=m_{\text {comfort }}\left\langle\|a(t+s)\|^{2}\right\rangle_{\mathrm{s}} .
$$

\section{FDM++: A Driver MOdEL BASED ON INSTANTANEOUS FUTURE RISK EXPECTATIONS}

\section{A. Combination of Future Expected Risk and Utility}

The total driving cost, including all risk sources $e$ and driving qualities $u$, is then given by the combination of the two contributions such that $C(t)=R_{\mathrm{INT}}(t)+Q_{\mathrm{INT}}(t)$ or, equivalently,

$$
C(t):=\sum_{e=1}^{N_{e}}\left\langle r_{e}(t+s)\right\rangle_{\mathrm{s}}+\sum_{u=0}^{N_{u}}\left\langle q_{u}(t+s)\right\rangle_{\mathrm{s}}
$$

given assumed predicted trajectories for all traffic participants (usually expressed as traffic participants state distributions with e.g. predicted means and variances as introduced in section III-A).

The first contribution $R_{\mathrm{INT}}(t)$ quantifies the currently (at time $t$ ) predicted risk costs given by the critical, disruptive events $e$. The second contribution $Q_{\mathrm{INT}}(t)$ quantifies the currently predicted other costs (penalties or benefits) which are not disruptive, but incur continuously during the traveled trajectory. These include driving benefits such as Eq. (32), and can easily be extended to describe general utility or penalty terms such as cruising speed adaptation, traffic lights and traffic rule violation fines, driving in potentially risky areas, targeted cruising velocity as well as driving comfort.

If we look at the total cost Eq. (35) in detail, in combination with the introduced definitions and derivations, the risk part is exactly the total expected, predicted riskrelated cost formulated in our risk model introduction in section II, Eq. (2), now expressed and calculated in terms of microscopic risk indicators $I_{e}$, instantaneous event rates $\tau_{e}^{-1}$, and the survival function $S(t+s, t)$.

\section{B. FDM++ Costs of Predicted Trajectories}

In the following, we combine all derived ingredients to sketch the full Foresighted Driver Model FDM++. For notational compactness, we do this for a scenario of an egovehicle with several other vehicles. We remark, however,
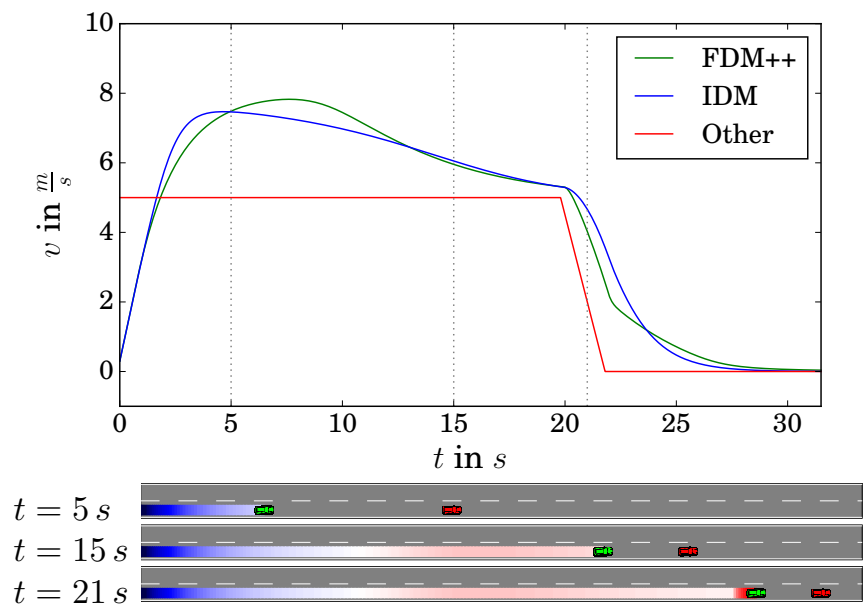

Fig. 2: Top: Speed profiles of a simple longitudinal scenario where the ego vehicle (green) starts with zero velocity, accelerates until it reaches its cruising velocity and brakes as reaction to a car ("other", red) in front. The risk prediction first leads to a smooth adaptation of the ego velocity to the velocity of the frontal car, and then to a foresighted braking. Comparison with IDM velocity profile (blue line). Bottom: Scenario snapshots with indicated acceleration / deceleration pattern.

that it applies analogously to scenarios with other items like pedestrians, static road elements, traffic signs and signals by modeling them with their appropriate risk indicator from section III and instantaneous event rate functions from section IV. We start at current time $t$ with a given, predicted ego-vehicle trajectory.

\section{Trajectory cost estimation}

- For each other traffic participant, predict its future state evolution in terms of probability distributions, described e.g. by trajectories $\hat{\mathbf{x}}(t+s)$ with variances $\sigma_{\mathbf{x}}(t+s)$ as introduced in section III-A.

- For each possible risk source $e$, calculate a risk indicator function $I_{e}(t+s)$ and the corresponding instantaneous future event rates $\tau_{e}^{-1}\left[I_{e}(t+s)\right]$ using the predicted states.

- With the instantaneous future event rates, calculate $\tau_{\text {tot }}^{-1}(t+s)$ and with that the survival function $S(t+s, t)$.

- For each risk event $e$, calculate the differential risk over predicted time $r_{e}(t+s)$.

- For each driving quality contribution $u$, calculate the differential cost over predicted time $q_{u}(t+s)$.

- With the survival function, the differential risk and driving quality costs, calculate the total driving cost $C(t)$.

\section{Driver Behavior as Instantaneous Optimization of Ex- pected Costs}

For behavior evaluation and planning, we assume that we have a dynamic ego-vehicle forward model with parameters $\theta(t)$ and constraints, which is used to calculate the predicted trajectory. E.g., longitudinally in addition to constant velocity 
prediction we may use a short acceleration or deceleration step to gain 3 slightly varied trajectories with parameters $\theta_{0}$, $\theta_{1}$ and $\theta_{2}$.

\section{FDM++ behavior selection}

- For ego-vehicle parameters $\theta_{n}$ variations do:

- Create predicted ego-vehicle trajectories from $\theta_{n}$.

- Calculate trajectory cost estimation $\Rightarrow C_{n}(t)$.

- Interpolate $C_{n}(t)$ as a function of $\theta_{n}(t) \Rightarrow \hat{C}(\theta, t)$.

- Choose $\hat{\theta}$ so that $\hat{\theta}(t)=\operatorname{argmin}_{\theta}[\hat{C}(\theta, t)]$ with $\hat{\theta}$ subject to the ego-vehicle model constraints

- (Optional) Weight $\hat{\theta}$ depending on the magnitude of the related costs.

- Use the behavior parameter $\hat{\theta}$ to execute, monitor or recommend an action.

- Advance a time step $\Delta t$ and repeat

Usually, for performance reasons the FDM++ loop is preceded by a preselection step where the algorithm is restricted to those traffic participants that have a chance to become relevant for interaction, e.g. by neglecting those that are to far or not on collision course with the ego vehicle.

The result is a behavior which seeks to instantaneously adjust its current driving parameter so that its impact on the future expected costs (in terms of risks and driving qualities) is optimal. The main rationale is that it is best to concentrate on the things that can be done immediately, rather than to expect benefits that lie far ahead in the future. This intuition is supported by the weighting via the survival function, which emphasizes the proximal over the distant future.

A full-blown optimization of the ego-vehicle predicted trajectory space is also feasible, and has been studied in numerous previous publications related to risk estimation and risk maps with survival theory, see e.g. [2]. Here, however, we introduced a simple and computationally cheap model which nevertheless is able to capture risks that occur during normal driving situations.

\section{Simulations}

In this section we show exemplary behaviors gained from the our FDM++ framework. We present a longitudinal frontal car following scenario, a speed adaptation scenario with two cars, and an overtaking speed adaptation scenario. The predicted trajectories of the other vehicles are assumed to have constant velocity, and we used 3 predicted ego-vehicle trajectories consisting of a short acceleration $a_{\max }$, no acceleration and a short deceleration $a_{\min }$ followed by constant velocity driving. In all scenarios we use the same parametrization. Where applicable, we show the corresponding IDM simulation. We used the parameter setting $\Delta t=0.1 \mathrm{~s}, T=1.6 \mathrm{~s}$, $s_{0}=2 m$ (IDM only), $a_{\max }=3 \mathrm{~m} / \mathrm{s}^{2}, a_{\min }=-3 \mathrm{~m} / \mathrm{s}^{2}$, $v_{\text {cruise }}=8 \mathrm{~m} / \mathrm{s}$ or variable (IDM and FDM++), $\tau_{0}^{-1}=3 / \mathrm{s}$, $\tau_{\max }^{-1}=10 / s$ (for collision risks), $\beta=5, m_{\text {cruise }}=0.001$, $m_{\text {comfort }}=0.0005, \sigma_{x}(t)=0.5 m$ (longitudinal, all vehicles), $\alpha_{v}=0.15, d_{\mathrm{long}, \text { min }}=4 m, d_{\mathrm{lat} \text {,min }}=2 m$ (FDM++ only). For the variation parameters we used $\theta_{n} \in\left[a_{\max }, 0, a_{\min }\right]$ and created 3 predicted ego-vehicle trajectories with a short acceleration, zero acceleration and deceleration step followed by constant velocity to calculate the costs $C_{n}(t)$.
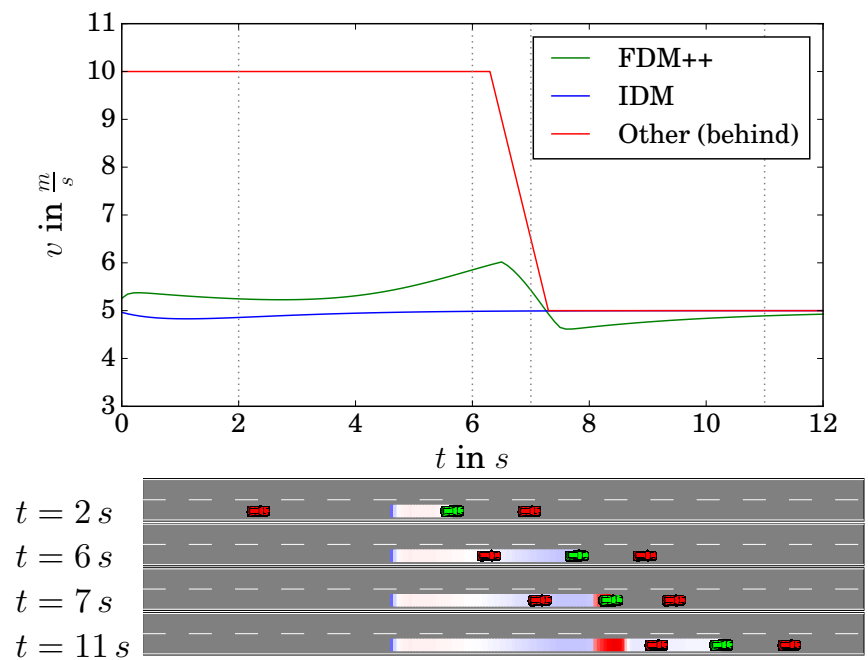

Fig. 3: The ego vehicle (green) is located between two other vehicles which narrow the gap. The frontal vehicle drives with constant velocity, the other one is far behind the ego vehicle $(t<2 s)$. The ego vehicle adjusts its velocity to the queue velocity at a gap position which minimizes both frontal and rear risk. At every moment, the FDM++ takes all relevant risk sources into consideration, estimating the total risk involved and leading to a reasonable risk-aversive behavior. Top: Speed profiles. Bottom: Scenario snapshots.

\section{A. Free Cruising and Frontal Vehicle Braking}

In this standard scenario, the ego vehicle starts with zero velocity on a quasi free road, where another vehicle drives with constant speed (see Fig. 2) at large distance. The FDM++ causes the ego vehicle to accelerate until it reaches its cruising velocity of $v_{\text {cruise }}=8 \mathrm{~m} / \mathrm{s}$ and then drive with constant velocity $(t=5 \mathrm{~s})$. Because of the higher velocity of ego compared to the other frontal car, the ego vehicle starts to decelerate continuously with decreasing vehicle distance $(t=$ $15 \mathrm{~s}$ ) because of an increase in the predicted collision risk. At some point, the ego-vehicle adapts to the same velocity as the other vehicle velocity, at a speed-dependent distance. When the frontal vehicle suddenly brakes until a standstill, the ego vehicle reacts by braking with maximal deceleration. For comparison, we simulated the same scenario based on the IDM. The behavior is qualitatively very similar, since both accelerate to cruising velocity and react to the sudden changing behavior.

\section{B. Gap adjustment with Aggressive Oncoming Driver}

The second scenario is a car following scenario where the ego vehicle is located between two other vehicles on a highway (see Fig. 3). The ego vehicle drives with constant velocity behind the frontal vehicle. A second vehicle approaches from behind with high constant velocity $(t<2 s)$. The ego vehicle now has two sources of risk to react to, considering the tailgating oncoming car and avoiding a crash with the frontal leading vehicle. The ego vehicle smoothly increases its velocity to mitigate the risk from the oncoming vehicle from behind $(t=6 \mathrm{~s})$. As a consequence, the relative 
velocity to the frontal vehicle increases and the ego vehicle has to find a tradeoff. At $(t=6.5 s)$, the oncoming vehicle reduces its velocity to the queue velocity, which relaxes the situation. The ego vehicle accelerates and takes its new distance to the frontal vehicle. In this case, the FDM++ considers several risk sources at once to react in a reasonable way.

\section{Narrow Overtaking}

The third is an overtaking scenario where the ego vehicle passes a vehicle in front at a small lateral distance, e.g. caused by a reduced street geometry as in construction zones. The lateral distance is sufficiently small so that the positional uncertainty estimation leads to a potential collision risk. The frontal vehicle is driving with constant velocity on the neighboring lane when the ego vehicle intends to overtake. To reduce the risk and the uncertainty of the lateral distance, the ego vehicle decreases its velocity prior to passing, until the moment of minimal distance is reached (at $t=5 \mathrm{~s}$ ). After passing, it accelerates back to cruising velocity, see Fig. 4 for 3 speed profiles for different cruising speed preference. Since the formulation of the FDM++ is able to incorporate longitudinal as well as lateral risks, it can be applied to scenarios like crossings, entrances and lateral passing as shown here in a straightforward way.

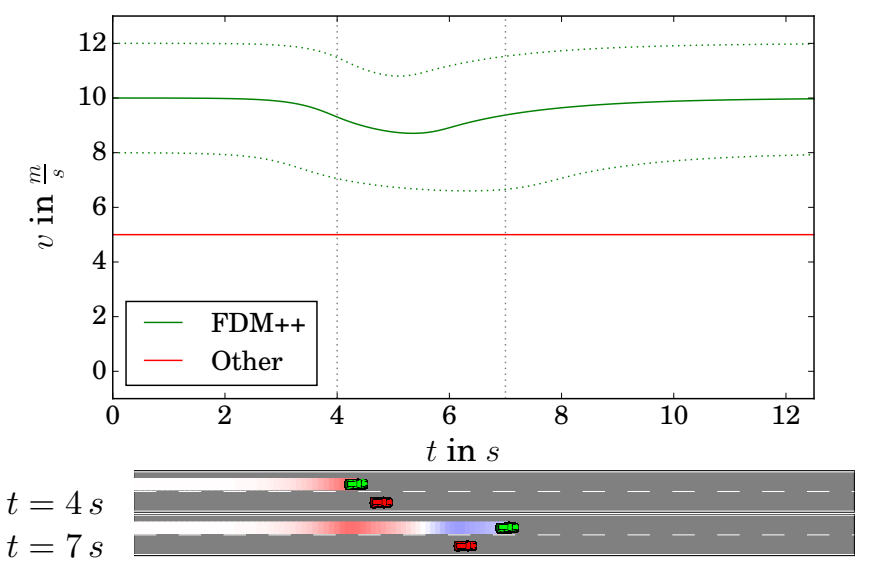

Fig. 4: Overtaking scenario: the ego vehicle (green) overtakes a car driving another car in front with small lateral distance. Positional uncertainty in the prediction of the other vehicle leads to a predicted collision risk which is reduced during the overtaking process by deceleration. The FDM++ adapts its behavior accounting for lateral in the same way as for longitudinal risks. Top: Speed profiles for 3 different cruising speed preferences. Bottom: Scenario snapshots of the $v_{\text {cruise }}=$ $10 \mathrm{~m} / \mathrm{s}$ speed preference.

\section{SUMMARY AND CONCLUSIONS}

Starting from a classical risk definition and a formulation of probabilistic, disruptive events, we have derived a compact driver model based on integral expected risk measures. A driver model then targets to find the behavior which minimizes these risk measures, finding a tradeoff between risk and driving benefits.

From its derivation, the model is able to incorporate different risk types, risk sources and risk events in a single formulation in a natural way. As shown in the simulations, this includes scenarios with longitudinal or lateral risks and several traffic participants. Detailed specification of some of the models contributions, such as the precise severity cost functions, as well as detailed parameter analysis are open for extensions and future investigations.

\section{REFERENCES}

[1] S. Lefèvre, D. Vasquez, and C. Laugier, "A survey on motion prediction and risk assessment for intelligent vehicles," ROBOMECH Journal, vol. 1, no. 1, p. 1, 2014. [Online]. Available: https://hal.inria.fr/hal-01053736

[2] T. Puphal, M. Probst, Y. Li, Y. Sakamoto, and J. Eggert, "Optimization of velocity ramps with survival analysis for intersection merge-ins," in 2018 IEEE Intelligent Vehicles Symposium (IV), June 2018, pp. 1704-1710.

[3] W. Xu, J. Pan, J. Wei, and J. M. Dolan, "Motion planning under uncertainty for on-road autonomous driving," in 2014 IEEE International Conference on Robotics and Automation (ICRA), May 2014, pp. 2507-2512.

[4] D. Ferguson, T. M. Howard, and M. Likhachev, "Motion planning in urban environments," Journal of Field Robotics, vol. 25, no. 11-12, pp. 939-960, 2008.

[5] X. Li, Z. Sun, D. Cao, Z. He, and Q. Zhu, "Real-time trajectory planning for autonomous urban driving: Framework, algorithms, and verifications," IEEE/ASME Transactions on Mechatronics, vol. 21, pp. 740-753, 2016.

[6] P. G. Gipps, "A behavioural car-following model for computer simulation," Transportation Research Part B: Methodological, vol. 15, no. 2, pp. 105-111, 1981.

[7] S. Krauß, "Towards a unified view of microscopic traffic flow theories," IFAC Proceedings Volumes, vol. 30, no. 8, pp. 901-905, 1997.

[8] M. Treiber, A. Hennecke, and D. Helbing, "Congested traffic states in empirical observations and microscopic simulations," Phys. Rev. E, vol. 62, pp. 1805-1824, Aug 2000. [Online]. Available: https://link.aps.org/doi/10.1103/PhysRevE.62.1805

[9] A. Kesting, M. Treiber, and D. Helbing, "General lane-changing model mobil for car-following models," Transportation Research Record, vol. 1999, pp. 86-94, 012007.

[10] M. Liebner, F. Klanner, M. Baumann, C. Ruhhammer, and C. Stiller, "Velocity-based driver intent inference at urban intersections in the presence of preceding vehicles," Intelligent Transportation Systems Magazine, IEEE, vol. 5, pp. 10-21, 062013.

[11] J. Eggert, F. Damerow, and S. Klingelschmitt, "The foresighted driver model," in 2015 IEEE Intelligent Vehicles Symposium (IV), June 2015, pp. 322-329.

[12] F. Damerow, B. Flade, and J. Eggert, "Extensions for the foresighted driver model: Tactical lane change, overtaking and continuous lateral control," in 2016 IEEE Intelligent Vehicles Symposium (IV), June 2016, pp. $186-193$.

[13] J. Eggert, "Predictive risk estimation for intelligent adas functions," in 17th International IEEE Conference on Intelligent Transportation Systems (ITSC). IEEE, Oct 2014, pp. 711-718.

[14] F. Damerow and J. Eggert, "Predictive risk maps," in 17th International IEEE Conference on Intelligent Transportation Systems (ITSC), Oct 2014, pp. 703-710.

[15] S. Luko, "Risk management principles and guidelines," Quality Engineering, vol. 25, 102013.

[16] J. Carrasco, S. Ferrari, and G. Cordeiro, "A new generalized kumaraswamy distribution," 042010.

[17] R. Garmier, B. Revelin, P. Legendre, S. Delavault, B. Vidal, and L. Lorda, "Computation of a collision probability based on a gaussian mixture model of the tle accuracy," 102009.

[18] A. Lambert, D. Gruyer, G. S. Pierre, and A. N. Ndjeng, "Collision probability assessment for speed control," in 2008 11th International IEEE Conference on Intelligent Transportation Systems, Oct 2008, pp. 1043-1048. 